\title{
DVR-BASED HYBRID ROUTING PROTOCOLS IN MOBILE AD-HOC NETWORK: APPLICATION AND CHALLENGES
}

\author{
Mrinal Kanti Debbarma ${ }^{1}$, Jhunu Debbarma ${ }^{2}$, Santanu Kr. Sen ${ }^{3}$, Sudipta Roy ${ }^{4}$ \\ ${ }^{1}$ Department of Information Technology, Assam University \\ ${ }^{2}$ Department of Information Technology, Assam University \\ ${ }^{3}$ Department of Computer Science \& Engg., BBIT, Kolkata \\ ${ }^{4}$ Department of Information Technology, Assam University
}

\begin{abstract}
DVR- hybrid routing protocol proposal derives from the conventional distance vector routing algorithm which, in turn, is based on Distributed Asynchronous Bellman Ford's shortest path algorithm, however, with some minor to significant variations as needed for the ad hoc and mobile nature of the MANET A major thrust of the protocol designers towards the development and deployment of DVR-based routing protocols, irrespectively in wired, wireless or even in ad hoc and sensor networks is primarily because of the conceptual and implementation simplicity and elegance coupled with the minimum information requirement by each node of the DVR. A good amount of research has been done in the past towards the improvement of routing algorithms in MANET, but the area has not become stable till date. This paper, focused on several applications, challenges and solutions, in the perspective of routing in MANET.
\end{abstract}

Keywords: MANET, Count-To-Infinity (CTI), Slow Convergence, Path Vector Routing (PVR).

\section{INTRODUCTION}

Mobile Ad-hoc network is a collection of mobile devices denoted as nodes, which can communicate between themselves using wireless links without the need or intervention of any infrastructure like base stations, access points etc [1][2][3]. A node in a MANET, which is equipped with a wireless transmitter and receiver (transceiver) and is powered by a battery, plays the dual role of a host and a router as well. Two nodes willing to communicate with each other need to be either in the direct common range of each other or should be assisted by other nodes acting as routers to carry forward the packets from a defined source to a destination in the best possible routing path [3,][4].

Internet Engineering Task Force (IETF) activity has standardized several routing protocols for MANET. Routing protocols are the backbone to provide efficient services in MANET, in terms of performance and reliability. Designing routing protocol in MANET is quite difficult and tricky compared to that of any classic or non-ad hoc (formal) network due to some inherent limitations of the MANET like dynamic nature of network topology, limited bandwidth, asymmetric links, scalability, mobility of nodes limited battery power and alike. Moreover, the intrinsic nature of the nodes to move freely and independently in any arbitrary direction by potentially changing ones link to other's on a regular basis, is really an exigent concern while designing the desired routing algorithm. MANET is IP based and the nodes have to be configured with a free IP address not only to send and receive messages, but also to act as router to forward traffic to some destination unrelated to its own use.

The main challenge to setup a MANET is that each node has to maintain the information required to route traffic properly and thus designing a routing protocol for MANET has several difficulties. Firstly, MANET has a dynamically changing topology as the nodes are mobile. However, this behavior favors routing protocols that dynamically discover routes (e.g. Dynamic Source Routing [5], TORA [6], Associativity Based Routing (ABR) [7] etc.) over conventional distance vector routing protocols (DVR) [5][6][8]. Secondly, the fact that MANET lacks any structure and thus makes IP subnetting inefficient. Thirdly, limitation of battery power and power depletion of nodes due to large number of messages passed during cluster formation. Links in mobile networks could be asymmetric at times. If a routing protocol relies only on bidirectional links, the size and connectivity of the network may be severely limited; in other words, a protocol that makes use of unidirectional links can significantly reduce network partitions and improve routing performance. DVRP is one of two major routing protocols for communications approach that use packets which are sent over IP. DVRP required routing how to report the distance of various nodes within a network or IP topology in order to determine the best and most efficient route for packets. 


\section{APPLICATIONS AND CHALLENGES}

The primary application of the MANET is in various field viz., battle field, disaster recovery, reuse operation, natural calamity, fast traffic information delivery on highways, network extension, local interconnection network, ubiquitous computing, sensor network, urban sensing, vehicular networking, home and enterprise networking, Wireless Local Area Network (WLAN), Private Area Network (PAN), health monitoring, tracking application, monitoring the aquatic environment, localization application, law enforcement, emergency service, educational application, conference/meeting/lectures, wildlife monitoring, intelligent home applications, urban areas, ubiquitous Internet access, delivery of location-wise information/service, entertainment, multiuser games, robotic pets and etc. However, a slight variation, namely wireless sensor networks (WSN), Vehicle Ad hoc Networks (VANET) which are ad hoc in nature, do possess wide applicability in the modern electronics world. Routing in such ad hoc networks possess a vital role in transmission of messages for what they are intended to. Routing is the heart of a network and optimality and reliability are the keen requirements for the success of such a routing protocol.

The important challenges in routing in MANETs are due to same intrinsic characteristic of MANETs, like limited battery power, highly dynamic nature of network topology, limited bandwidth, asymmetric links, scalability, mobility of nodes etc

Recent challenges for ad-hoc wireless networks comprise:

- Multicast [4]

- QoS support

- Power-aware routing [5]

- $\quad$ Location-aided routing [6]

Multicast is appropriate to support cooperative /multiparty wireless communications. The multicast tree is highly dynamic in nature (i.e., its topology is subject to change over time), the multicast routing protocol must be able to handle with mobility, including multicast membership dynamics (e.g., join and leave). In case of QoS, it is insufficient to consider QoS simply at the network level without considering the underlying MAC layer [7]. Again, as nodes are dynamic in nature, the problems associated with the hidden station, and inconsistent link characteristics, supporting end-to-end QoS is a nontrivial issue that involves in-depth analysis and research. In recent years, there is a trend toward an adaptive QoS approach as a substitute of the "plain" resource reservation method with hard QoS guarantees. An additional important factor is the limited power source in handheld nodes, which can seriously forbid packet forwarding in an ad hoc mobile environment. Therefore, routing traffic based on nodes' power metrics is one way to differentiate routes that are more prolonged than others. Finally, as a substitute of using beacon or broadcast search Location-Aided Routing (LAR) uses positioning information to define associated regions at the routing is spatially concerned with.
This is comparable to associativity-oriented and restricted broadcast in ABR.

\section{DVR BASED ROUTING PROTOCOLS IN MANETS: CLASSIFICATIONS}

The Distance Vector Routing Algorithm (DVRA) is a dynamic (adaptive), distributed (decentralized), asynchronous and iterative routing protocol where the routing tables are continuously updated with the information received from the neighbouring routers [2], [7]. The DVRA requires each router to periodically compute its shortest path (best route) to every other router in the network, exchange this distance information in the form of a DVT, with its neighbours and thereby update its own routing table (DVRT). This DVRT updation process is continued till a stable DVRT is obtained which the router can then use to forward packets to other routers. Among the other routers in the network, some are its neighbours who are connected to it either by a direct point-to-point link or via any broadcast subnets like Ethernet or Token Ring. The shortest path or best route often implies the minimum-delay path. Periodically, but not synchronously with other routers, each router measures its distance to each neighbouring router by sending time-stamped ECHO REQUEST packets to them and receiving time-stamped ECHO RESPONSE packets from them. It then updates its DVRT using the Distributed Asynchronous Bellman-ford Algorithm [7] and checks if any change has occurred. In case any change occurs, it builds an updated DVT from this updated DVRT and sends this updated DVT to all its neighbouring routers. Finally, it starts using its updated DVRT for routing packets. Similarly, it receives, in turn, and, asynchronously, the periodically updated DVTs sent by each of its neighbours.

Before proceeding farther, it should be expressly pointed out that, unlike a DVT which a router sends to and receives from its neighbours, a DVRT is for the router's own use and each entry in the DVRT has one additional column (parameter) compared to those in the DVT, namely, the identity of the next-hop, i.e., the neighbouring router that provides this (shortest path) route. Now, upon receiving a regular or expected DVT from a neighbour, corresponding to the Periodic Update (PU) carried out by the latter, a router recomputed its own DVRT. In case any change occurs in its DVRT, the router immediately sends its correspondingly changed DVT to all its neighbours. Receipt of this updated DVT triggers the neighbours to immediately recompute their respective DVRTs. If this triggered update (TU) brings about any change in the DVRT of any of the neighbours, the latter immediately sends its updated DVT to all its neighbours which may, in turn, result in further TUs. This additional DVT exchange process (besides the periodic exchange process) continues till no TU yields any further change in the DVRT of any router. The DVRA is then said to have converged, i.e., attained a stable state. 
DVR is a simple, elegant and widely used as the Internet Gateway Protocol (IGP) and Extended Gateway Protocol (EGP) [3] in the Global Internet as well as in the private Internets in the form of Routing Information Protocol (RIP)[2,3], RIP-2 and Boarder Gateway Protocol (BGP)[2,3]. The Distance Vector Routing Algorithm (DVRA) is a dynamic, distributed, asynchronous and iterative routing protocol where the routing tables are continuously updated with the information received from the neighbouring routers [2], [10].

As a distributed dynamic routing algorithm which is expected to adapt to changes in topology and traffic. The existing DVRA, though simple and conceptually elegant, suffers from some well-known problems like Count-to-Infirmity (CTI), slow convenience, looping, frequent route oscillation. Out of all these, the CTI is more vulnerable, which actually, made practical DVR out of race.

Though good amount of research has been done in the past towards the improvement of the basic DVRA [18], none of the proposed techniques like Hold-Down, Split Horizon and Poison Reverse have been able to satisfactory solve the problems in the DVRA.

DVRA is used in routing protocols similar to IGRP [18], ISO and Novell IPX due to its simplicity, elegance and flexibility. We have investigated a modified DVRA (MDVRA), which uses a host of novel techniques to remove all the weaknesses, particularly the CTIP, inherent to the existing DVRA. Additionally, the goal is to augment the existing capabilities of the DVRA so as to make it efficient, robust, fully dynamic and fully fit to the environment of the MANET primarily and WSN in the extended phase.

\section{CHALLENGES OF DVR}

There are some weaknesses of the asynchronous DVRA are as follows:

- Count-To-Infinity (CTI) Problem

- Slow Convergence Problem

- Oscillation Problem

\subsection{Count-To-Infinity (CTI) Problem}

The DVRA cannot perform fine if there are topological changes in the internetwork. DVRA suffers from CTI problem due to link or router failure or due to unending routing loops. The CTI caused by routing loops involving more than two routers are providing in [8].

\subsection{Slow Convergence (SC) Problem}

When there is growth in terms of cost of any link or there is a failure in the link between two corresponding neighboring nodes in an internetwork, then it considered as worst case or requires unnecessary number of iterations to terminate.

\subsection{Oscillation Problem}

Besides CTI and slow convergence the another important problem of the DVR or compatible shortest path routing protocols is that because of the need to always use the shortest path, there may be frequent switching of routes caused by even small increase or decrease in the link costs. This frequent route switching gives rise to instability in routing and this problem is known as the route oscillation problem [17]. If two paths to a destination have delays which are marginally different, then the choice of the lower delay path as the shortest path invites more traffic on this path so that it ceases to be the shortest path in no time and, as a result, the other path becomes shorter than this path. As a consequence, more traffic now flows through the second path, thus increasing its delay and makes it lose the status of the shortest path to the original shortest path. The route oscillation problem degrades overall performance of a network and hence some mechanism is needed to dampen the oscillation, i.e., to reduce the frequency of route switching. It should be noted in this context that if hop count is used as the metric in the DVR, then the route oscillation problem does not arise at all because the traffic changes which cause changes in the link delays are ignored.

\subsection{Existing Solutions to the CTI Problem in the DVRA}

In the global Internet, the DVR is used as an intra- domain routing protocol [14], i.e., it can be used inside an autonomous system (AS), but it is not suitable for inter-domain routing, mainly because of the CTI problem. The Path Vector Routing (PVR) [14], which is also derived from the DVR, is used for inter-domain routing. In the PVR, there is at least one node, known as speaker node, in each AS that acts on behalf of the entire AS. The speaker node in an AS creates a routing table and advertises it to speaker nodes in the neighboring AS. The idea is the same as for the DVR except that only speaker nodes in each AS can communicate with the speaker nodes in the neighbouring AS. However, what is advertised is different. A speaker node advertises its full AS path for reaching every destination AS. Just as in the DVR, an AS in the PVR shares its table with only its immediate neighbours.

The instability of the DVR and the possibility of the creation of loops are avoided in the PVR because it advertises the full path to a destination, instead of only the distance (number of AS hops). When a router receives a route update message, it checks to see if its own AS is in the path list to the destination. If it is, the message is ignored and thus looping is avoided. Border Gateway Protocol (BGP) [13], [17], which is the de-facto standard for inter domain routing protocol in the Internet is based on the PVR and avoids looping and CTI problem. However, advertisement of the full path in the BGP entails large overhead (BGP is a complex routing protocol having many other attributes besides path vector which also requires large overhead) [14],[20],[21]. On the other hand, although BGP is 
based on the DVR, the actual implementation of BP has moved much away from the DVR. The simplicity and elegance of DVR, which it is famous for, is ignored and overlooked in BGP and the other PVR-based routing protocols.

\subsection{Distributed Routing Approach}

Distributed Routing adapts a compromise between the two extreme approaches adapted by the centralized routing (a fully global but centralized approach) and isolated routing (a purely local approach) by sharing the knowledge about the network gained by other routers. This makes Distributed Routing perform much better than the other two dynamic routing algorithms and, as a result, most of today's routing algorithms are distributed in nature.

Among the distributed routing algorithms, two are most widely used, namely the Distance Vector Routing Algorithm (DVRA) which is based on the well-known Bellman-Ford shortest path algorithm and the Link State Routing Algorithm (LSRA) which is based on the well-known Dijkstra's shortest path algorithm. In the DVRA, each router periodically exchanges, with its neighbouring routers, its Distance Vector Table (DVT) containing entries specifying the identity of each known router (destination) along with the current estimated distance of the shortest path to reach the router. Utilizing the DVTs received from all its neighbours and measuring its own distance to each neighbour, a route computes the shortest path distance to each destination, builds its own Distance Vector Routing Table (DVRT) and sends the DVT (unlike the DVRT, the DVT does not contain the next-hop information in the entries).The manuscript should be prepared in accordance with the reviewer's comments after having the final acceptance. BGP has been derived from the DVR protocol. However, BGP differs from the DVR protocol in at least two important ways. First, instead of just advertising its distance in total number of hops to a certain destination, the AS advertises its complete AS-path upto the destination. For this reason BGP is also called a path vector protocol. Second, the path or route selection in the BGP is based on not only the shortest path criterion but also the "import" and "export" policies that may be independently chosen by each router [13] [14].

\section{CONCLUSIONS}

The problems of routing in MANETs and other such ad hoc networks like wireless sensor networks (WSN), Vehicle Ad hoc Networks (VANET), etc are primarily because of the mobility of the nodes, random topology changes, limited battery power, limited bandwidth, asymmetric links, frequent link breakage due to random addition and deletion of nodes, to name a few, for which, many routing protocols already proposed and/or in research, have not found to be stable and thus providing enough scope of improvement through research and investigations.

In this paper, focused on several applications, challenges and solutions, in the perspective of routing in MANET and many routing schemes based on DVR in the said field have been investigated and compared with each other keeping technical relevancy, as far as practicable. The review shows that a majority class of existing routing protocols is fundamentally DVR based as because of the conceptual and implementation simplicity and elegancy of the DVR. In this paper, a DVR-based routing algorithm for MANETs has been investigated and suggested keeping stress on the existing solution to the CTI problem of DVRA. The key focus is made on application and challenges with proper identification of solution, so that each neighbour can provide more and more information of different nature to its adjacent router and thus each router could enrich its information database by collecting such information from all of its next-hop neighbours, which would help during routing traffic proactively or reactively as the situation demands.

\section{REFERENCES}

[1] Albeto Leon-Garcia and Indra Widjaja, Communication Networks, Tata McGraw Hill, 2000

[2] N. H. Saeed, M.F. Abbod, and H S. AI Raweshidy, "MANET Routing Protocols Taxanomy", IEEE International Conference on Future Communication Networks, PP-123-128, 2012

[3] M. Abolhasan et al. " A review of routing protocols for mobile ad hoc networks" Elsevier Ad Hoc networks 2 (2004) $1-22$

[4] M. Gerla, C.C Chiang, and 1. Zhang, "Tree Maulticast Strategies in Mobile, Multihop Wireless Networks," ACM/Baltzer Mobile Networks and Apps. J,. 1988

[5] S. Singh, M. Woo, and C. S. Raghavendra, "PowerAware Routing in Mobile Ad Hoc Networks," Proc. ACM/IEEE MOBICOM '98, Oct. 1998.

[6] Y. B. Ko and N. H. Vaidya, "Location-Aided Routing (LAR) in Mobile Ad Hoc Networks," Proc. ACM/IEEE MOBICOM '98, Oct. 1998.

[7] S. Das, C. Perkins, E. Royer, Ad hoc on demand distancevector (AODV) routing, Internet Draft, draftietf-manetaodv-11.txt, work in progress, 2002.

[8] S. Murthy J.J. Garcia-Luna-Aceves, A routing protocol for packet radio networks, in: Proceedings of the First AnnualACM International Conference on Mobile Computing andNetworking, Berkeley, CA, 1995, pp. 8695.

[9] C. C. Chiang, T. C. Tsai, W. Liu, and M. Gerla, "Routing in clustered multihop, mobile wireless networks with fading channel," The Next Millennium, Proceedings of IEEE Singapore International Conference on Networks, SICON, pp. 197-221, 1997.

[10] G. Pei, M. Gerla, X. Hong, and C. C. Chiang, "A wireless hierarchical routing protocol with group mobility," IEEE Wireless Communications and Networking Conference, WCNC '99, vol.3, pp. 1538 1542, New Orleans, LA, 1999.

[11] P. Jacquet, P. Muhlethaler, T. Clausen, A. Laouiti, A. Qayyum, L. Viennot, Optimized link state routing 
protocolfor ad hoc networks, IEEE INMIC, Pakistan, 2001.

[12] M. Gerla, Fisheye state routing protocol (FSR) for ad hoc networks, Internet Draft, draft-ietf-manet-aodv03.txt,work in progress, 2002.

[13] T.-W. Chen, M. Gerla, Global state routing: a new routing scheme for ad-hoc wireless networks, in: Proceedings of theIEEE ICC, 1998.

[14] T. Griffin and G. Wilfong, " A safe Path Vector Protocol" Proc. IEEE INFOCOM, vol. 2, March 2000, pp-490-499.

[15] C. Toh, A novel distributed routing protocol to support ad- hoc mobile computing, in: IEEE 15th Annual International Phoenix Conf., 1996, pp. 480-486.

[16] S. Tenenbaum, Computer Networks, 3rd Ed., PHI, 2000.

[17] Douglas E. Comer, David L. Stevens, Internetworking with TCP/IP Vol-II, 3rd Ed., Pearson Education (Singapore), LPE, 2005

[18] D. Bertsekas and R. Gallagar: "Data Networks", 2nd Ed., Englewood cliffs, NJ, Prentice Hall, 1992

[19] J. F. Kurose and K. W. Ross, Computer Networking: A Top-Down Approach Featuring the Interent, Third Edition, Pearson Education, Inc, Singapore, 2005.

[20] S. K. Ray, J. Kumar, S. K. Sen and J. Nath, "Modified Distance Vector Routing Scheme for a MANET", Proc. Of the 13th National Conference on Communications (NCC) held at IIT, Kanpur during Jan 26-28, 2007, pp. 197-201.

[21] G.V. Kumar et al. "Current Research Work on Routing Protocols for MANET: A Literature Survey" International Journal on Computer Science and Engineering, Vol. 02, No. 03, 2012, 706-713 